\title{
Dark-matter test faces obstacles
}

A group of scientists is hoping to replicate a controversial Italian experiment that claims to have detected dark matter. But they might have to do so without the help, or the equipment, of the original group.

Dark matter is thought to make up around $85 \%$ of the matter in the Universe, but it rarely interacts with regular matter except through the force of gravity. Researchers working on the DAMA experiment at the Gran Sasso National Laboratory near L'Aquila, Italy, claim they have spotted direct signs of it.

The detector used by the DAMA team consists of 250 kilograms of ultrapure sodium iodide crystals placed 1,400 metres beneath Gran Sasso mountain. Over the past decade, the researchers have collected data showing that nuclei in the crystals periodically release flashes of light, which could be caused by interactions with dark matter. Crucially, the number of flashes varies with the seasons, which would be consistent with Earth's motion through a galactic dark-matter stream (R. Bernabei et al. Eur. Phys. J. C 56, 333-355; 2008).

But other detectors have so far failed to see an effect, leading some to conclude that DAMA's signal is the result of radioactive contamination inside the sodium iodide crystals. "There are very good reasons to disbelieve the signal," says Adam Falkowski, a theoretical physicist at Rutgers University in Piscataway, New Jersey.

Still, Frank Calaprice at Princeton University in New Jersey says that the signal is significant enough to be followed up. "It could be right - they're careful people," he says of the DAMA team. "I

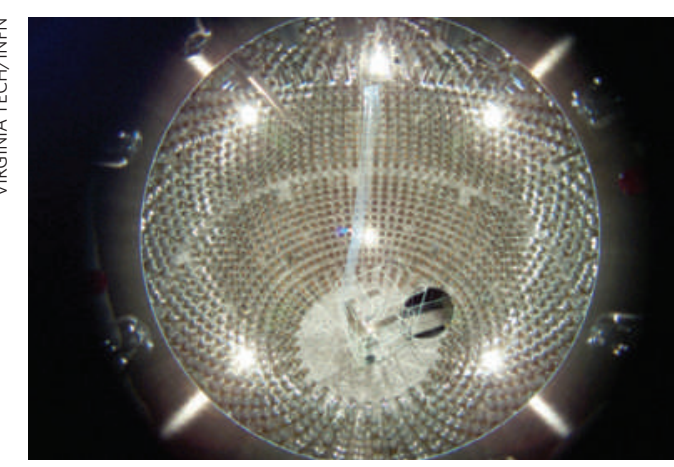

The Borexino experiment may start looking for dark matter as well as solar neutrinos. think it deserves to be checked."

Calaprice and some of his colleagues believe that building a similar detector out of sodium iodide is the only way to verify DAMA's claim definitively. They want to place those crystals inside another Gran Sasso experiment, called Borexino, which uses 300 tonnes of a liquid organic compound to detect solar neutrinos - nearly massless particles that stream from the Sun. Detecting neutrinos requires careful control of background signals, which makes Borexino the perfect place for checking whether DAMA's crystals really are seeing dark matter, says Calaprice, a Borexino team member.

DAMA's principal investigator disagrees. The proposal "is not good for many scientific reasons", says Rita Bernabei, a physicist at the National Institute of Nuclear Physics in Rome. "Borexino cannot ensure the fulfilment of all the stringent requirements necessary for a reliable measurement in this field," she says.

Bernabei adds that no one at Borexino has formally asked DAMA for help in setting up another sodium iodide crystal detector, and declined to say whether such help would be provided if asked.

Reproducing DAMA's experiment without its cooperation is difficult because an intellectual-property agreement between the group and its supplier, Saint-Gobain in Paris, prevents the sale of the ultrapure sodium iodide crystals to other groups. A Spanish experiment known as ANAIS, located at the Canfranc underground laboratory near Zaragoza, Spain, has already been prevented from buying the crystals. "We have had to spend time and extra money to search for an alternative," says José-Angel Villar of the University of Zaragoza, who is leading the collaboration. With the help of an American supplier, he says the group hopes to begin checking DAMA's findings soon.

Calaprice says he is hopeful that his team's test can go ahead, and is investigating the possibility of growing his own crystals. The group's proposal will have to be submitted to the full Borexino collaboration, which will decide whether to take it forward. Geoff Brumfiel 